OPEN ACCESS

Edited by:

Mikhail Lebedev,

Duke University, USA

Reviewed by:

Dongrui WU,

University of Southern California, USA Aleksandra Vuckovic

University of Glasgow, UK

Anastasios Bezerianos,

National University of Singapore,

Singapore

*Correspondence:

Pietro Aricò

pietro.arico@uniroma1.it

${ }^{\dagger}$ These authors have contributed equally to this work.

Received: 01 July 2016 Accepted: 11 October 2016 Published: 26 October 2016

Citation:

Aricò P, Borghini G, Di Flumeri G, Colosimo A, Bonelli S, Golfetti A, Pozzi S, Imbert J-P, Granger G, Benhacene R and Babiloni F (2016)

Adaptive Automation Triggered by EEG-Based Mental Workload Index: A

Passive Brain-Computer Interface Application in Realistic Air Traffic Control Environment.

Front. Hum. Neurosci. 10:539. doi: 10.3389/fnhum.2016.00539

\section{Adaptive Automation Triggered by EEG-Based Mental Workload Index: A Passive Brain-Computer Interface Application in Realistic Air Traffic Control Environment}

\author{
Pietro Aricò ${ }^{1,2,3 * t}$, Gianluca Borghini 1, 2,3t, Gianluca Di Flumeri ${ }^{2,3,4 \dagger}$, Alfredo Colosimo ${ }^{4}$, \\ Stefano Bonelli ${ }^{5}$, Alessia Golfetti ${ }^{5}$, Simone Pozzi ${ }^{5}$, Jean-Paul Imbert ${ }^{6}$, Géraud Granger ${ }^{6}$, \\ Raïlane Benhacene ${ }^{6}$ and Fabio Babiloni ${ }^{1,2}$

\begin{abstract}
${ }^{1}$ Department of Molecular Medicine, Sapienza University of Rome, Rome, Italy, ${ }^{2}$ BrainSigns Co. Ltd, Spin-off Company from Sapienza University of Rome, Rome, Italy, ${ }^{3}$ Neuroelectrical Imaging and BCI Lab, Fondazione Santa Lucia (IRCCS), Rome, Italy, ${ }^{4}$ Department of Anatomical, Histological, Forensic Medicine and Orthopedic Sciences, Sapienza University of Rome, Rome, Italy, ${ }^{5}$ DeepBlue srl, Rome, Italy, ${ }^{6}$ École Nationale de l'Aviation Civile, Toulouse, France
\end{abstract}

Adaptive Automation (AA) is a promising approach to keep the task workload demand within appropriate levels in order to avoid both the under- and over-load conditions, hence enhancing the overall performance and safety of the human-machine system. The main issue on the use of $A A$ is how to trigger the $A A$ solutions without affecting the operative task. In this regard, passive Brain-Computer Interface $(\mathrm{pBCl})$ systems are a good candidate to activate automation, since they are able to gather information about the covert behavior (e.g., mental workload) of a subject by analyzing its neurophysiological signals (i.e., brain activity), and without interfering with the ongoing operational activity. We proposed a $\mathrm{pBCl}$ system able to trigger AA solutions integrated in a realistic Air Traffic Management (ATM) research simulator developed and hosted at ENAC (École Nationale de l'Aviation Civile of Toulouse, France). Twelve Air Traffic Controller (ATCO) students have been involved in the experiment and they have been asked to perform ATM scenarios with and without the support of the AA solutions. Results demonstrated the effectiveness of the proposed $\mathrm{pBCl}$ system, since it enabled the AA mostly during the high-demanding conditions (i.e., overload situations) inducing a reduction of the mental workload under which the ATCOs were operating. On the contrary, as desired, the AA was not activated when workload level was under the threshold, to prevent too low demanding conditions that could bring the operator's workload level toward potentially dangerous conditions of underload.

Keywords: passive brain-computer interface (pBCl), Adaptive Automation (AA), Air Traffic Management (ATM), electroencephalogram (EEG), mental workload, human factors, machine learning, human machine interaction 


\section{INTRODUCTION}

The main goal of Human Factor (HF) studies is to ensure good interactions between the work environment and human capabilities (Wickens, 1992). Humans can adapt themselves to a variety of work environments, performing various tasks, also simultaneously, by using different equipment. Obviously, the greater the number and variety of tasks to perform and devices to use is, the higher the workload experienced is. It has been widely demonstrated that too high operator's mental workload level (overload) could result in a degradation of performance and/or an increase in the errors commission probability (Reason, 2000). Therefore, during the past decades, it has been deeply investigated the possibility of developing intelligent systems able to automatically support the operator in executing its working tasks, in order to reduce the experienced workload, and consequently keep the performance within high levels, and limit the probability of errors commission.

In general, the term "Automation" refers to the process of entirely or partially allocating activities constituting a task usually performed by a human, to a machine, or a system (Parsons, 1985). In particular, Sheridan (1992) identified 10 different Level of Automation (LOA), differing in the number and type of activities of the whole task allocated to the operator and to the system, from the Level 1 (operator's fully manual control) to the Level 10 (fully automated control). The first developed form of automation was the Static Automation (St-A), i.e., an on/off technology active at a fixed LOA, or not active at all. Certainly, the introduction of St-A in operational environments has brought clear benefits (Scerbo, 1996), however research on human interactions with automation has shown that St-A could also introduce several disadvantages, such as monitoring inefficiency, loss of situational awareness, impaired decision making, complacency, and manual skill degradation (Parasuraman et al., 1993). In contrast, systems in which automated aids are implemented dynamically, in response to changing task demands on the operator, may be less vulnerable to such problems. This kind of automation is called Adaptive Automation (AA) (Rouse, 1976), recently described as one of the "most important ideas in the history of HF and ergonomics" (Hancock et al., 2013). In particular, an AA-based system is able to adjust continuously the proper LOA, i.e., to assign the authority on specific functions to either the humans or the automated system, depending on the task difficulty and the operator's workload. It has been demonstrated how Adaptive Automation is superior to Static Automation, since the former is able to ensure operator's workload within the optimum range, preserve his/her skill level, guarantee continuous task involvement, and vigilance, thus increasing his/her performance (Rouse, 1988; Wickens, 1992; Byrne and Parasuraman, 1996). Several strategies concerning the triggering mechanism for shifting among modes or levels of automation have been proposed (Scerbo et al., 2003). Three are the main approaches described in the literature: (i) the Critical-event strategy, based on the a-priori assumption that human workload may become too high when the critical events occur (Hilburn et al., 1997); (ii) the Performance-measurement strategy, based on the use of operator's performance during the task itself or additional ones (also called behavioral measures) to estimate current and predicted operator's state and to infer whether workload is excessive or not; (iii) Neurophysiological measurement strategy, based on the recording of operator's neurophysiological signals, e.g., electroencephalogram (EEG), electrocardiogram (ECG), Galvanic Skin Response (GSR), to infer his actual mental workload (Scerbo et al., 2001). Collectively, each strategy has pros and cons. Although the use of the first two approaches has been successfully employed in several studies, the neurophysiological measurements-based approach has several advantages (Byrne and Parasuraman, 1996). Firstly, unlike the critical-events approaches, neurophysiological measures could be obtained continuously and online. Secondly, compared with performance measures, the neurophysiological ones may be recorded continuously without using overt responses (i.e., additional tasks) and may provide a direct measure of the mental (covert) activities of the operator. Also, neurophysiological measures have higher resolution than performance measures (Di Flumeri et al., 2015). Finally, neurophysiological measures can be used not only to trigger the AA, but also to highlight why AAs are important for enhance the safety in high-risk and high-demanding tasks. This potentially offers new perspectives for adaptive intervention to optimize performance by acting on specific aspects of the operator's behavior.

Byrne and Parasuraman (1996) assessed that the advantage of applying neurophysiological measures in triggering AA was very clear, but the "effective application of psychophysiology in the regulatory role may require years of effort and considerable maturation in technology." Nowadays, 20 years later, such "effective application" could become reality thanks to the progresses in Brain-Computer Interfaces (BCI) research. Briefly, a BCI is defined as "a system that measures Central Nervous System (CNS) activity and converts it into artificial output that replaces, restores, enhances or improves natural CNS output and thereby changes the ongoing interactions between the CNS and its external or internal environment" (Wolpaw and Wolpaw, 2012). Such definition summarizes the progresses of the scientific community in this field during the last decades, since at the moment the possibility of using the BCI systems outside the laboratories (Aloise et al., 2010; Blankertz et al., 2010; Aricò et al., 2011; Riccio et al., 2015; Schettini et al., 2015), by developing applications in everyday life is not just a theory but something very close to real applications (Zander et al., 2009; Blankertz et al., 2010; Aricò et al., 2016). This technology has been defined passive BrainComputer Interface (pBCI). In particular, in pBCI technologies, the system recognizes the spontaneous brain activity of the user related to the considered mental state (e.g., emotional state, workload, attention levels), and uses such information to improve and modulate the interaction between the operator and the system itself. Thus, in the context of AA, the pBCIs perfectly match the needs of the system in terms of Human-Machine Interaction (Parasuraman et al., 1992; Zander and Jatzev, 2012).

In this context, the most studied mental state is the Mental Workload (MWL), due to its strong relationship with the user's performance variations. MWL is a complex construct, generally defined as the actual task cognitive demand related to the real cognitive capacity of the operator (O'Donnell and Eggemeier, 
1986). Several empirical investigations have suggested that performance declines at either far ends of the workload demand profile, i.e., when event rates are excessively high (overload) or extremely low (underload) (Yerkes and Dodson, 1908; Calabrese, 2008). Therefore, it is crucial to have a reliable estimation of the actual mental workload experienced by the operator along the execution of the task, in order to make the user interface able to preserve a proper level of the user's mental workload, avoiding under- or overload state (Hancock and Warm, 1989; Borghini et al., 2012, 2015b). In this regard, neurophysiological techniques have been demonstrated to be able to assess mental workload of humans with a high reliability, even in operational environments (Mühl et al., 2014; Borghini et al., 2015a; Di Flumeri et al., 2015). Many neurophysiological measures have been used for the mental workload assessment, including Electroencephalography (EEG), functional Near-InfraRed (fNIR) imaging, functional Magnetic Resonance Imaging (fMRI), and other biosignals, such as Electrocardiography (ECG) and Galvanic Skin Response (GSR) (Wood and Grafman, 2003; Ramnani and Owen, 2004; Borghini et al., 2014). Among all these techniques, Aricò et al. (2016) have highlighted the clear advantages of using EEG signal to implement pBCI applications. Several studies, in particular in the aviation domain, have developed efficient EEG-based mental workload indexes. The preliminary results of Brookings et al. (1996) showed that the effects of the task demand were evident on the EEG rhythms variations. EEG power spectra increased in the theta band, while significantly decreased in the alpha band as the task difficulty increased, over parietal and frontal brain sites. More recently, Shou et al. (2012) found that "the frontal theta EEG activity was a sensitive and reliable metric to assess workload [...] during an ATC task at the resolution of minute (s)." The same findings have been highlighted by Borghini et al. (2013) involving pilots in flight simulation tasks. In other recent studies involving ATCOs (Aricò et al., 2013, 2014, 2015b,c; Borghini et al., 2014; Di Flumeri et al., 2015; Toppi et al., 2016), it was demonstrated how it was possible to compute an EEG-based Workload Index able to significantly discriminate the workload demands of the ATM task, and to monitor them continuously by using frontal-parietal brain features. Other studies about the mental workload estimation by using neurophysiological indexes, have been proposed also in other operational contexts (Car drivers - Kohlmorgen et al., 2007; Borghini et al., 2012a; military domain - Dorneich et al., 2005).

Despite the scientific evidences on the possibility of measure the mental workload by using neurophysiological measures, and of using them to trigger AA solutions (i.e., pBCI), only few examples have been proposed in this regard, the most of them in laboratory settings. The concept of a "closed-loop system," i.e., the mitigation of an operator's level of workload through a closedloop system driven by the operator's own EEG, was theorized during the past decade (Prinzel et al., 2000; Schmorrow et al., 2006). Freeman et al. (1999) proposed one of the first EEG-based studies about the impact and efficiency of AA: they developed an application able to switch between automatic and manual mode of the tracking task of the Multiple-Attribute Task Battery (MATB, Comstock, 1994) by adopting EEG indexes based on the Theta and Alpha band power spectra over the parietal cortex, according to the results obtained in a precedent study (Pope et al., 1995). A similar study was also proposed by Prinzel et al. (2000). Both the studies highlighted a significant decrement in the mental workload experienced by the users by activating the automation solutions, confirmed by using both the EEG-based indexes and the subjective measures. Also, Berka et al. (2005) studied a similar application by using the Aegis simulator, i.e., a military simulation environment. Also in this study it has been highlighted the possibility of monitoring in real-time the mental workload and to use EEG-based indexes to reallocate tasks and system aids. However, as stated before, such studies were performed in laboratory settings. Recently, Abbass et al. (2014) built an adaptive controller's working position based on cues extracted from EEG signals and task complexity indicators from the scenario, demonstrating that four operators achieved a better performance while the AA was activated. Apart from such preliminary studies, no evidences about the possibility to use $\mathrm{pBCI}$ technologies to realize AA systems in real settings have been proposed.

In this study, we present a passive-BCI system fully integrated with a high realistic ATM simulator able to trigger adaptive solutions in real-time depending on the mental workload estimated by means of the ATCO's brain activity. We expected that the pBCI system would be able to trigger the AA solutions and to reduce the task workload demand in order avoid both under and overload conditions.

\section{MATERIALS AND METHODS}

\section{Subjects}

Twelve Air Traffic Controller (ATC) students ( $23 \pm 2$ years old) from the École Nationale de l'Aviation Civile (ENAC, Toulouse, France, one of the most important training schools for ATCOs and Pilots in the World) have been involved in this study. They were selected in order to have a homogeneous experimental group in terms of age and expertise. These students were finishing their 3 years training at ENAC. The experiment was conducted following the principles outlined in the Declaration of Helsinki of 1975 , as revised in 2000. It received the favorable opinion from the Ethical Committee of the Sapienza University of Rome, Dept. Physiology and Pharmacology. The study involved only healthy, normal subjects, recruiting on a voluntary basis. Subjects were free to accept or not to take part to the experimental protocol. All the recruited subjects accepted to participate to the study. Informed consent was obtained from each subject on paper, after the explanation of the study. No other individual information apart from the cerebral activity was gathered for the purpose of this study. Only aggregate information has been released while no individual information were or will be diffused in any form.

\section{Experimental Protocol}

The subjects have been asked to manage a functional interface that simulates a high realistic ATM scenario. The complexity of the task could be modulated according to how many aircrafts the ATCO had to control, the number and type of clearances required over the time and the number/trajectory of other interfering flights. Also, for the purposes of this study, specific 
AA solutions have been embedded in the ATM interface, with the aim to induce a decreasing in the operators' mental workload during high workload situations. These AA solutions were the result of several brainstorming sessions with subject matters experts (senior ATCOs), human factor and human computer interaction specialists. According to design principles described in the Introduction Section, few realistic proposals have been made and implemented. Those AA solutions have been described in Table 1.

Such ATM interface has been developed and hosted at ENAC. Electroencephalogram (EEG) signal has been recorded and used online to evaluate the mental workload of the controllers. Such mental state has been used to trigger the RADAR screen interface by using the AA solutions described previously, only when the workload of the user become higher than the threshold defined during a specific calibration phase. Triggers depending on the actual mental workload of the user has been sent to the ATC interface by using a dedicated middleware developed at ENAC. Figure 1A shows the platform architecture realized for the purpose of such experiment.

ATCO students that took part to the experiment were already well trained to use such interface. In particular, controllers have been asked to handle with two ATM scenarios under two different conditions (Easy and Hard). One scenario in which the AA could be triggered by the EEG-based mental workload index of the user $(A A O n)$, and the other one in which the operators' EEG-based mental workload index has been computed and stored, but not used to trigger the ATM interface (AAOff). Each scenario lasted 15 ( $\mathrm{min}$ ), the first 5 and the last 5 ( $\mathrm{min}$ ) have been designed to keep the task difficulty as constant as possible, low (Easy), and high (Hard), respectively. The middle part of the task (5 min) has been designed to simulate a realistic transition between the Easy and Hard segments, but it has not been used in the analysis (Figure 1B). The two scenarios have been designed comparable in terms of complexity within the same difficulty levels (e.g., Easy of Scenario 1 and Easy of Scenario 2). The combinations of scenarios and conditions (AA On/Off) have been randomized to avoid any habituation effect and bias in the results. In addition, ATCO students have been asked to perform two traffic samples of 3 ( $\mathrm{min}$ ) before the execution of the experimental scenarios, respectively, easy (Easy 0), and hard (Hard 0), to be used for the calibration of the EEG-based workload algorithm. Let us name such tasks of 3 ( $\mathrm{min}$ ) as "Calibration scenarios" and the two consecutive 15 min-long scenarios as "Testing scenarios." At the end of each Testing scenario, Controllers have been asked to fill the NASA-TLX questionnaire (Hart and Staveland, 1988), in order to evaluate their perceived mental workload in performing the different conditions (AA On/Off). NASA-TLX is a widely-used, multidimensional assessment tool that rates subjective perceived workload between 0 and 100 .

\section{EEG-Based Online Mental Workload Classifier Signals recording}

For each subject, scalp EEG signal was recorded (g.USBamp, gTec, Austria) from $9 \mathrm{Ag} / \mathrm{AgCl}$ wet electrodes (Fpz, Fz, F3, F4, AF3, AF4, Pz, P3, P4) at $256(\mathrm{~Hz})$, referenced to both the mastoids and grounded to the $\mathrm{Cz}$ electrode, according to the 10-20 International System (Jurcak et al., 2007).

\section{Processing}

The recorded EEG signal has been band-pass filtered $(1 \div 30$ $(\mathrm{Hz})$, 5th order Butterworth filter) and the Fpz channel has

TABLE 1 | Description of the AA solutions developed at ENAC.

\begin{tabular}{|c|c|}
\hline AA solution & Description \\
\hline \multirow[t]{3}{*}{$\begin{array}{l}\text { Adapt Situation Awareness Monitoring by reducing or } \\
\text { removing alerts }\end{array}$} & $\begin{array}{l}\text { The monitoring agent sends all alerts to the controllers not considering the controller's workload, traffic } \\
\text { complexity or the alert emergency. The interface could filter those alerts to prevent distracting the controller } \\
\text { with an alert which is not critical if the controller's workload is high. }\end{array}$ \\
\hline & High workload: Only critical alarms are shown to the controller. \\
\hline & Low workload: No alarms \\
\hline \multirow[t]{3}{*}{ Highlighting of calling station } & $\begin{array}{l}\text { Aircraft labels on the radar image are highlighted to help controllers locate the aircraft currently speaking on } \\
\text { the radio. }\end{array}$ \\
\hline & $\begin{array}{l}\text { High workload: the background of the classing of a calling station is blue and it remains as it is until the } \\
\text { controller moves the mouser pointer over the aircraft. }\end{array}$ \\
\hline & Low workload: no highlight \\
\hline
\end{tabular}

Adapt Short Term Collision Avoidance (STCA) alert design
The graphical design of the STCA is not the most efficient to catch controller's attention. The design could be changed to alert the controller faster. An animated box around the label will reduce the perception time of the controller.

High workload: graphical design used is box animation (a box appears around the label with some margin and shrinks until no margin is left)

Low workload: graphical design used is color blinking 


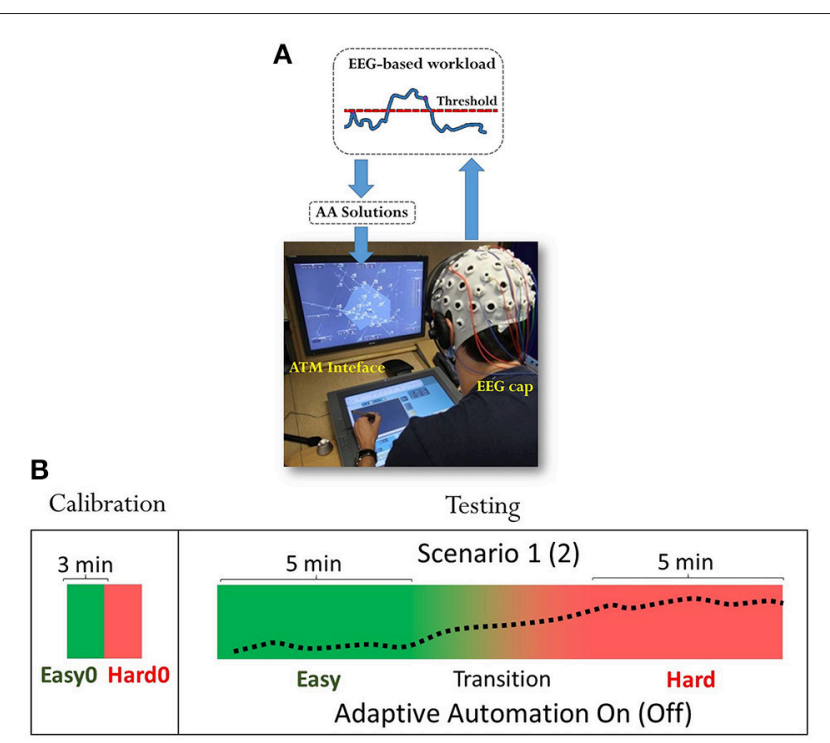

FIGURE 1 | (A) figure shows ATCO students wearing the EEG cap during the experiment and managing the ENAC platform, composed of two screens, a $30^{\prime \prime}$ (RADAR) screen to display radar image and a $21^{\prime \prime}$ screen to interact with the radar image (ATM interface). The mental workload of the user was evaluated online and specific AA solutions changed online the behavior of the RADAR screen depending on the actual mental workload level. (B) ATCO students have been asked to perform two ATM scenarios, one in which adaptive solutions could be triggered by the EEG mental workload index (AA On), and the other one in which adaptive automation has been disabled (AA Off). Presentation of each scenario and condition has been randomized to avoid any habituation and expectation effects.

been used to remove eyes-blink artifacts from the EEG data by using the regression-based algorithm REBLINCA (Di Flumeri et al., in press). With respect to other regressive algorithms (e.g., Gratton method, Gratton et al., 1983) the REBLINCA algorithm has the advantages to preserve EEG information in blink-free signal segments by using a specific threshold criterion that recognize automatically the occurrence of an eye-blink, and only in this case the method correct the EEG signals. If there is not any blink, the method has not any effect on the EEG signal. In addition, the REBLINCA method does not require EOG signal(s). The band-pass filtered $(1 \div 7(\mathrm{~Hz})$, 5th order Butterworth filter) Fpz signal has been used as template to remove eye-blinks contribution from the EEG signal. Regressive weights for each EEG channel were calculated on the Calibration scenarios and have been used both offline (in the same scenarios) and even online in the Testing scenarios. This step has been performed because the eye-blinks contribution could affect the frequency bands related to the mental workload, in particular the theta EEG band. At this point, the EEG signal has been segmented into epochs of 2 (s), shifted of 0.125 (s). For other sources of artifacts (i.e., ATCOs normally communicate verbally and perform several movements during their operational activity), specific procedures (Threshold criterion, Trend estimation, Sample-to-sample difference) available in the EEGLAB toolbox (Delorme and Makeig, 2004) have been applied. In particular, in the Threshold criterion the EEG epochs are marked as "artifact" if the EEG amplitude is higher than $\pm 100(\mu \mathrm{V})$. In the trend estimation, the EEG epoch is interpolated in order to check the slope of the trend within the considered epoch. If such slope is higher than 3 ( $\mu \mathrm{V} / \mathrm{epoch}$ ), the considered epoch will be marked as "artefact." The last step calculates the difference between consecutive EEG samples. If such difference, in terms of amplitude, is higher than $25(\mu \mathrm{V})$, it means that an abrupt variation (no-physiological) happened, thus it will be marked as "artefact." Hence, the epochs marked as "artifact" were totally rejected.

\section{Algorithm calibration}

As stated before, the Calibration scenarios (Easy 0 and Hard 0) have been used to calibrate the algorithm before the Testing scenarios presentation. In particular, the Power Spectral Density (PSD) of EEG epochs related to each calibration scenario (Easy 0 and Hard 0 ) has been calculated by using only the frequency bands directly correlated to the mental workload (frontal theta and parietal alpha bands). The EEG frequency bands [frequency resolution of $0.5(\mathrm{~Hz})$ ] of interest have been defined for each ATCO by the estimation of the Individual Alpha Frequency (IAF) value (Klimesch, 1999; Babiloni et al., 2000). At this point, the classification algorithm automatic stop Stepwise Linear Discriminant Analysis (asSWLDA, patent number P1108IT00, Aricò et al., 2015a, 2016) has been used to identify the most relevant discriminant features among the different experimental conditions (i.e., Easy 0 and Hard 0), related to the lowest and the highest task complexity. Once identified, the asSWLDA classifier assigns to each significant feature specific weights $\left(w_{i}\right.$ train $)$, plus a bias $\left(b_{\text {train }}\right)$. On the contrary, weights related to those features not relevant for the classification model are set to " 0 ." These parameters have been used later on to compute online the mental workload index of the user during the Testing scenarios.

\section{StepWise Linear Discriminant Analysis (SWLDA)}

The SWLDA regression consists in the combination of the forward and the backward stepwise analyses, where the input features are weighted by using ordinary least-squares regression to predict the target class labels. The method starts by creating an initial model of the discriminant function in which the most statistically significant feature is added to the model for predicting the target labels $\left(\right.$ pval $_{i j}<\alpha_{\text {ENTER }}$ ), where pval ${ }_{i j}$ represents the $p$-value of the ith feature at the jth iteration (in this case the first iteration). Then, at each new iteration, a new term is added to the model (if pval ${ }_{i j}<\alpha_{\text {ENTER }}$ ). If there are not more features that satisfy this condition, a backward elimination analysis is performed to remove the least statistically significant feature (if $\mathrm{pval}_{\mathrm{ij}}>\alpha_{\mathrm{REMOVE}}$ ) from the model. This process goes on unless there are no more features satisfying the entry $\left(\alpha_{\text {ENTER }}\right)$ and the removal $\left(\alpha_{\text {REMOVE }}\right)$ conditions (Draper, $1998)$, or until a predefined number of iterations is reached

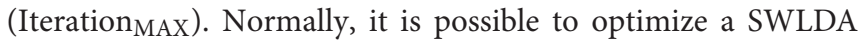
regression by tuning all or some of the three parameters available in the algorithm $\left(\alpha_{\text {ENTER }}, \alpha_{\text {REMOVE }}\right.$, and Iteraction $\left.n_{\text {AXX }}\right)$. There are not standard procedures to choose these parameters, and in theory, they should be easily manually (empirically) gauged based on the expected characteristics of the data. 
The standard SWLDA algorithm uses $\alpha_{\text {ENTER }}=0.05$ and $\alpha_{\text {REMOVE }}=0.1$, and no constrains on the Iteraction MAX $_{\text {MA }}$ parameter are imposed. For summarize, the standard training process goes on unless there are no more features satisfying the entry $\left(\alpha_{\text {ENTER }}\right)$ and the removal ( $\left.\alpha_{\text {REMOVE }}\right)$ conditions.

\section{Automatic stop StepWise Linear Discriminant Analysis (asSWLDA)}

The SWLDA is one of the best outperforming linear classifiers (Craven et al., 2006; Aloise et al., 2012), in fact with respect to other linear methods it has the advantage of having automatic features extraction, so that insignificant terms are statistically removed from the model. Despite the strength of the method, it is simple to realize how it would be difficult to set up properly the right parameters in order to optimize the algorithm. In fact, it is expected that the more general the classification training would be, the higher the reliability of the algorithm over time will be (Vapnik, 2000). For example, if the chosen parameters are too selective $\left(\alpha_{\text {ENTER }}\right.$ and/or $\alpha_{\text {REMOVE }}$, and/or Iteration $n_{M A X}$ values too much low), maybe the features added to the model will be not sufficient for predicting the target labels (underfitting, von Luxburg and Schoelkopf, 2011). On the contrary ( $\alpha_{\text {ENTER }}$

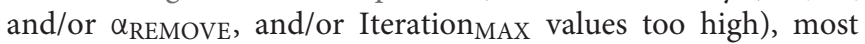
of the features added in the final model could be related to spurious differences between classes of the training set, that are obviously not generalizable, so that, the reliability of the algorithm decreases over time (overfitting, Vapnik, 2000). The reduction of features selected by the classifier in general could mitigate the overfitting.

The optimum solution to these problems would be to find out a criterion able to automatically stop the algorithm iterations when the best number of features (\#Features OPTIMUM) have been added to the model. In the following, it will be reported a modified version of the standard SWLDA algorithm that encloses this "automatic stop" criterion described previously. The name of this implementation is automatic-stop Stepwise Linear Discriminant Analysis (asSWLDA, Aricò et al., 2015a, 2016).

As we stated above, the tuning parameters in the standard SWLDA algorithm are three: $\alpha_{\text {ENTER }}, \alpha_{\text {REMOVE }}$, Iteration ${ }_{\text {MAX }}$. In this implementation, the first two parameters are left unbind as in the standard SWLDA implementation (i.e., $\alpha_{\mathrm{ENTER}}=0.05$, $\alpha_{\text {REMOVE }}=0.1$ ). In fact, because of the probability $\left(\right.$ pval $\left._{\mathrm{ij}}\right)$ associated to each feature is strictly related to the actual iteration (in other words, to all the actual features in the models) this probability changes iteration by iteration, and it would result very difficult to impose a condition by using $\alpha_{\text {ENTER }}$ and $\alpha_{\text {REMOVE. }}$. In addition, even if no constrains on the $\alpha_{\text {ENTER }}$ and the $\alpha_{\text {REMOVE }}$ parameters are imposed, the features would be included in the model in order of significance (i.e., the first feature in the model will be the most significant one, and so on). On the contrary,

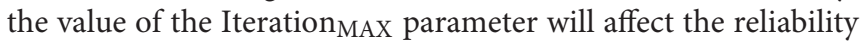
of the classifier over time (optimum classifier, underfitting, or overfitting). As we stated before, this parameter should be chosen such that \#Features UNDERFITTING $<<$ \#Features OPTIMUM $<<\#$ Features OVERFITting. In order to make the classifier able to find automatically the best IterationMAX parameter, we took into account the $p$-value of the model (pModel), parameter available in the output of the standard SWLDA implementation, that gives information about the global significance of the model at the iteration $\mathrm{jth}$. The more the number of iterations increases (the more features are added to the model), the more the pModel value decreases (tending to zero) with a decreasing exponential shape. First of all, we collected the pModel values for all the iterations [pModel(\#iter), Figure 2A]. At this point, we calculated the $\log 10$ of the pModel vector $\left[\log _{10}\right.$ [pModel(\#iter)], Figure 2B] , and then the first-order differences between adjacent pModel elements (Equation 1) that we called Convergence function, or Conv(\#iter). We used the $\log 10$ function since we would have information about the size of pModel order, and after that about the differences between these pModel orders.

Finally, we plotted this vector as a function of \#iter (Figure 2C).

$$
\begin{aligned}
\text { Conv }(\# \text { iter })= & \log _{10}(\text { pModel }(\# \text { iter }+1)) \\
& -\log _{10}(\text { pModel }(\# \text { iter }))
\end{aligned}
$$

We identified as the best Iteration ${ }_{M A X}$ 's value the number of iterations at which the Conv(\#iter) assumed the lowest distance from the point $(0,0)$, plus one (because we are working on the first-order differences, Formulas 2, 3).

$$
\begin{aligned}
\| \operatorname{Conv}\left(\# \text { iter }_{B E S T}\right) \| & =\min \| \operatorname{Conv}(\# \text { iter }) \| \\
\text { Iteration }_{M A X} & =\# \text { terer }_{B E S T}+1
\end{aligned}
$$

In fact, the best condition would be to have the least possible features and at the same time the convergence of the model (Formula 4).

$$
\log _{10}(\text { pModel }(\# \text { iter }+1))-\log _{10}(\text { pModel }(\# \text { iter }))=0
$$

Online EEG-based workload index ( $\left.W_{E E G}\right)$ assessment: The procedures described above have been applied ongoing with the execution of the testing scenario. To summarize, the band-pass filtered EEG signal has been buffered online in 2 (s) epochs shifted of 0.125 (s). EEG epochs have been cleaned off by eye-blinks contributions and those affected by other sources of artifacts have been discarded. At this point, PSD of the remaining EEG epochs have been calculated by using frontal theta and parietal alpha bands.

Thereafter, the classifier parameters estimated during the calibration phase $\left(\mathrm{w}_{i}\right.$ train and $\left.\mathrm{b}_{\text {train }}\right)$ have been used to calculate online the Linear Discriminant Function [ $y_{\text {test }}(t)$, Equation 5], defined as the linear combination of the testing spectral features [PSD calculated by using frontal $\theta$ and parietal $\alpha$ bands, $f$ test $(t)$ ] and the classifier weights $\left(w_{i}\right.$ train $)$, plus the bias $\left(b_{\text {train }}\right)$.

Finally, a moving average of $n$ seconds (nMA) has been applied to the $y_{\text {test }}(t)$ function in order to smooth it out by reducing the variance of the measures, and the result has been named EEG-based workload Index $\left(\mathrm{W}_{E E G}\right.$, Equation 6). The higher is the $n$ value, the less the variance of the measure will be. For a proper evaluation of the mental workload during the execution of ATM tasks, the $n$ value has been set to 30 (s). This value has been chosen together with ATCO and human factor experts, in order to find out the best trade-off between providing 


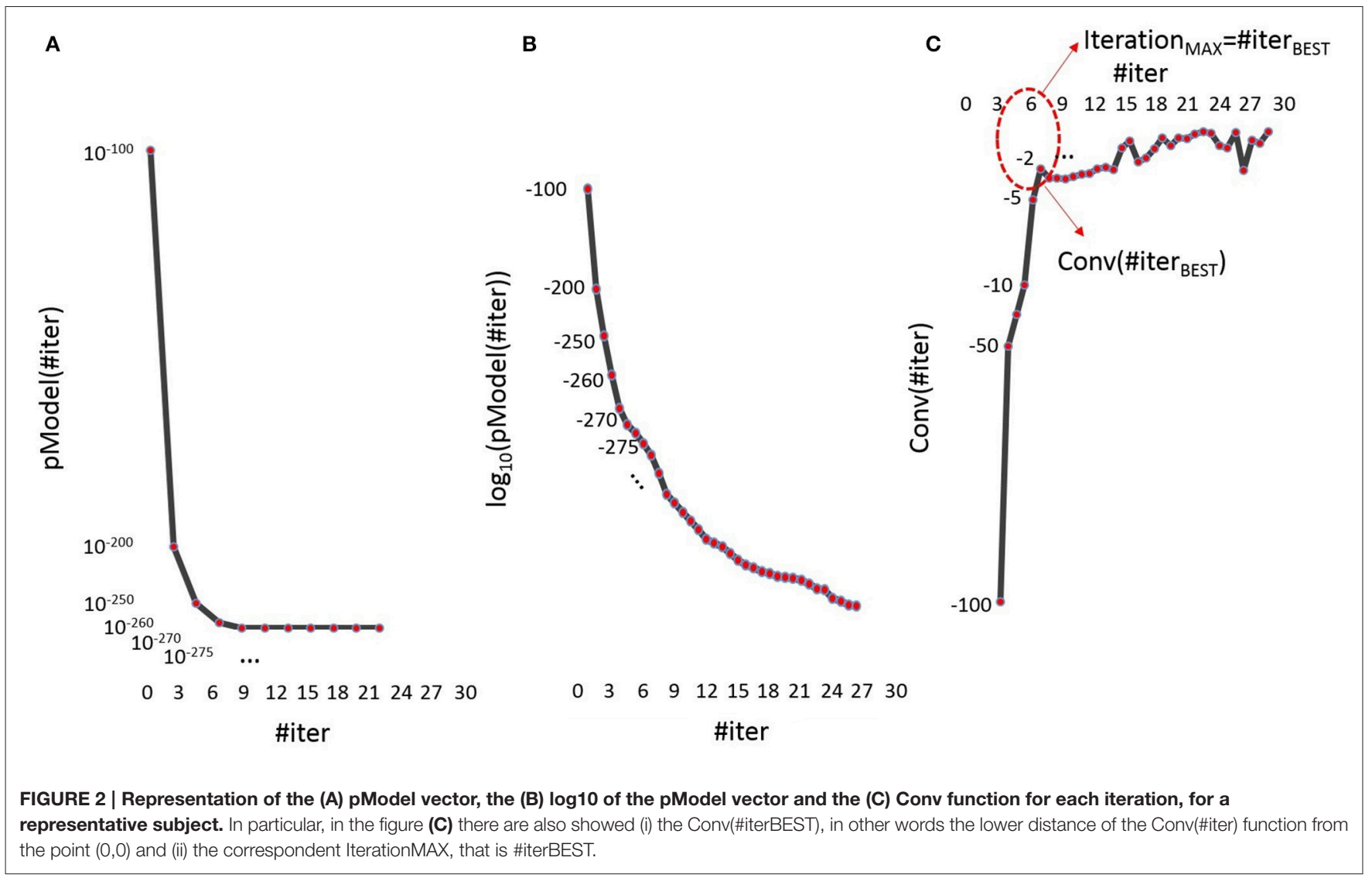

a proper workload resolution and, at the same time, an adequate commutation rate of the AA. In addition, in literature it has been shown that EEG is a viable source of information regarding the workload of a person, enabling 95\% accuracy when using about 30 s of signal (Gevins et al., 1998).

$$
\begin{aligned}
y_{\text {test }}(t) & =\sum_{i} w_{i_{\text {train }}} * f_{i_{\text {test }}}(t)+b_{\text {train }} \\
W_{\text {EEG }} & =n M A\left(y_{\text {test }}(t)\right)
\end{aligned}
$$

$\mathrm{i}=\#$ of spectral features; $\mathrm{t}=[1,2, \ldots, \#$ of EEG epochs $] ; \mathrm{n}=30(\mathrm{~s})$.

Online $W_{E E G}$ classification: The mental workload index $\left(\mathrm{W}_{E E G}\right)$ has been classified online in two classes (HIGH and LOW) to trigger the AA. In particular, if the $\mathrm{W}_{E E G}$ was higher than a specific threshold, the mental workload of the user was classified as HIGH and the adaptive solutions would be activated. On the contrary, the adaptive solutions were disabled (LOW class). In this regard, a proper connection has been created with the ATM interface, by using the TCP/IP network standard protocol in order to exchange messages (e.g., time frame of the experiment, workload index, classification result, etc.) in realtime. The classification threshold (Class-Threshold) was obtained through a procedure that relies on the use of Receiver Operating Characteristic (ROC) curves (Bamber, 1975). This methodology allows to estimate the performance of a binary classifier as its threshold is varied, by representing the true positive rate (TPR) against the false positive rate (FPR, Bamber, 1975). In this regard, for each subject a $k$-fold cross-validation $(k=10$, McLachlan et al., 2005) has been performed on the spectral features dataset related to the Calibration scenarios [PSD calculated by using frontal theta and parietal alpha bands, $\left.f^{j}(t)\right]$. In particular, all the possible (k-1) subsamples have been used to train the asSWLDA classifier, and the related parameters $\left(w_{i}^{j}, b^{j}\right)$ have been applied on the remaining subsample to compute the Linear Discriminant Function [ $y^{j}(t)$, Formula 7]. All the $y^{j}(t)$ values related to the $k$ iterations, have been stacked in the $Y$ vector (Formula 8).

$$
\begin{aligned}
y^{j}(t) & =\sum_{i} w_{i}^{j} * f_{i}^{j}(t)+b^{j} \\
Y & =\bigcup_{j} \bigcup_{t} y^{j}(t)
\end{aligned}
$$

$\mathrm{i}=\#$ of spectral features; $\mathrm{j}=[1,2, \ldots, \mathrm{k}] ; \mathrm{t}=[1,2, \ldots, \#$ of $\mathrm{EEG}$ epochs].

This vector $(Y)$ and the related labels vector (containing information if the specific element of the $Y$ vector is related to the Easy0 or Hard0 calibration scenario) have been used to compute the ROC curve. To select the best threshold (Class-Threshold) we used the "minimum distance" method, explained in details in Fawcett (2006). In other words, we selected as Class-Threshold for each subject the $Y$ value such that the FPR had been as low as possible, and at the same time the TPR as high as possible.

In the following, it has been reported the graphical representation of the averaged ROC over all the subjects related to the offline $k$-fold cross-validations performed to find the best 


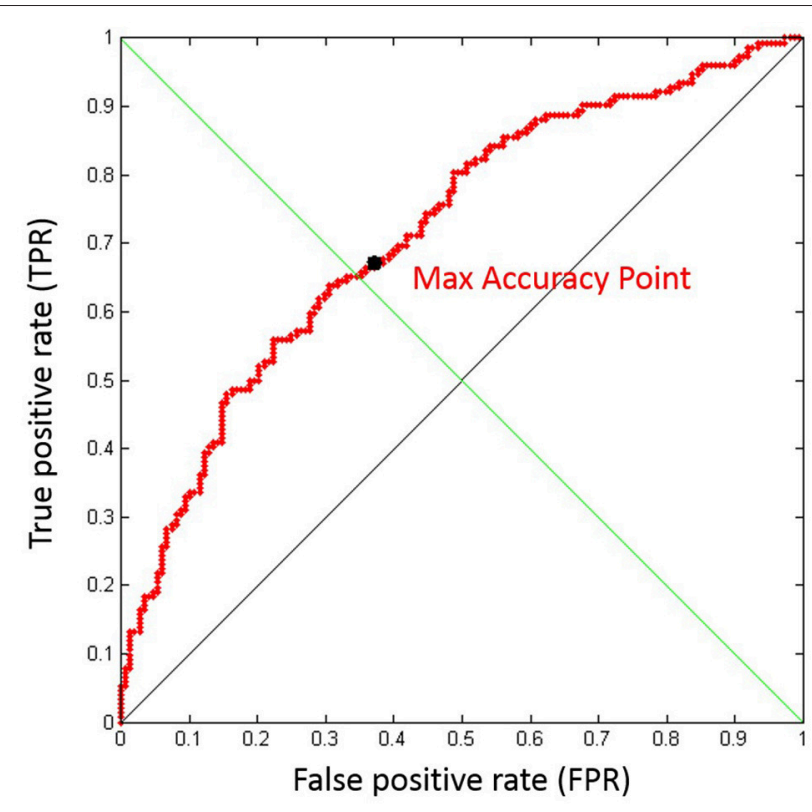

FIGURE 3 | Graphical representation of the averaged Receiver Operating Characteristic (ROC) over all the subjects related to the offline k-fold cross-validations performed to find the best threshold. The achieved offline classification performance was of $75 \pm 10 \%$. The mean threshold value was $0.48 \pm 0.07$

threshold. The mean offline classification accuracy achieved was $75 \pm 10 \%$. The mean threshold value was $0.48 \pm 0.07$ (Figure 3).

\section{Performed Data Analyses}

The following analyses had the aim to demonstrate the two hypothesis of the present study:

1. it is possible to trigger the AA by using the online recognition of the actual mental workload of the user,

2. the AA induces a reduction of the mental workload of the operator when it became high and consequently an increasing in performances execution of the task.

\section{Triggering of adaptive solutions}

To demonstrate the first hypothesis, we compared the number of times in which the $\mathrm{W}_{\mathrm{EEG}}$ activated the adaptive solutions in the Easy and Hard slots during the $A A O n$ condition, by using a two-tailed paired $t$-test $(\alpha=0.05)$. In fact, we expected that the mental workload classifier was able to induce an activation of the AA more often during the Hard period with respect to the Easy one.

To investigate the second hypothesis, we performed analyses on subjective and neurophysiological workload measurements along the two conditions (AA On/Off) and difficulty levels (Easy and Hard), and behavioral performances achieved by the operators in the two conditions.

\section{Subjective workload assessment}

A two-tailed paired $t$-test $(\alpha=0.05)$ has been performed on the NASA-TLX scores to investigate differences between the two AA conditions (AA On/Off) in terms of workload perception.

\section{Behavioural performance assessment}

The ATM interface recorded information about the reaction time and the number of airplanes the ATCO had assumed in each specific task condition (AA On/Off). Together with ATCO and human factor experts we defined an index based on these parameters related to the performance achieved by the operator during the execution of the task. In the following all the considered parameters used to assess the performance of the operator have been reported:

- Time Shoot: the interaction time needed for delivering an aircraft to the next sector,

- Time Route: the interaction time to display the graphic route of an aircraft,

- Time Cancel: the interaction time between triggering write recognition box and pressing of the cancel button,

- Time Annul: the interaction time between the opening of a pie menu or write recognition box and clicking on the radar image background,

- Time Turn: the interaction time between triggering the menu and the validation of the write recognition box,

- Time Flight Level: the interaction time between triggering the menu and the validation of the write recognition box,

- Time Direct: the interaction time between triggering the menu and selecting the waypoint in the flight plan list.

Since the ATCOs might adopt different strategies to manage the air-traffic, their reaction times have been normalized on the number of airplanes assumed in the different phases of the simulation. The performance index, Weighted Mean Reaction Time (WMRT), has then been defined as the average of the weighted reaction times described previously.

A two-tailed paired $t$-test $(\alpha=0.05)$ has been performed between WMRT indexes over the two conditions (AA On/Off).

\section{Neurophysiological workload assessment}

We compared the $\mathrm{W}_{E E G}$ indexes referred to the two difficulty levels (Easy, Hard) within and between the two AA conditions (AA On/Off). In particular, we performed 4 two-tailed paired $t$-test $(\alpha=0.05)$ to compare difficulty levels within the same AA condition (i.e., AA On: Easy vs. Hard; AA Off: Easy vs. Hard), and to compare the two AA conditions within the same difficulty level (Easy: AA On vs. AA Off; Hard: AA On vs. AA Off).

Before every statistical analysis, the $z$-score transformation (Zhang et al., 1999) has been used to normalize the data.

\section{RESULTS}

\section{Subjective Workload Assessment Analysis}

The $t$-test results showed that the perceived workload of the user during the $A A O f f$ condition was not significantly higher $(p=0.068)$ in comparison to the $A A$ On condition. It has to be underlined that the workload scores provided by the subjects referred to the whole AA condition, composed by the Easy, the Hard and the transition portion (Figure 4A). 
A

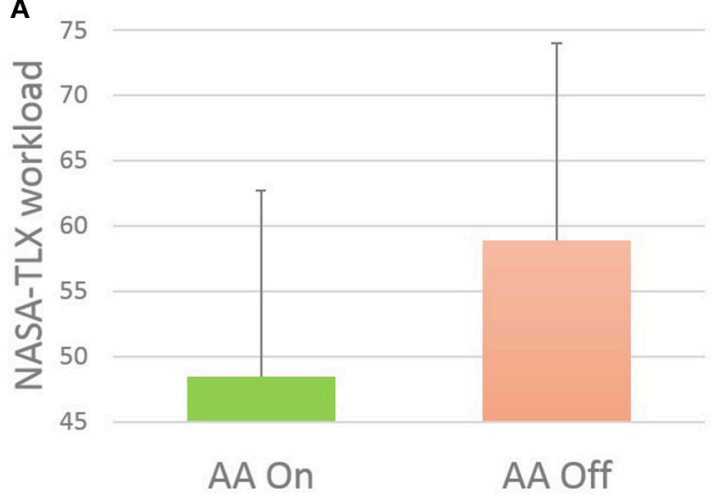

B

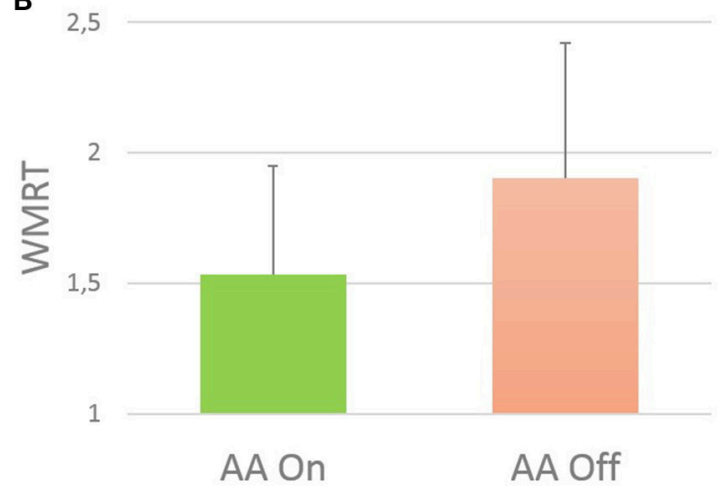

FIGURE 4 | (A) Vertical bars related to the subjective measure of the mental workload of the ATCOs, by using the NASA-TLX questionnaire. The results showed a not significant trend $(p=0.068)$ between the two conditions (AA On and AA Off). (B) Vertical bars related to the Weighted Reaction Time index (WMRT), reflecting behavioral performances of operators during the two conditions (AA On and AA Off). The results showed a significant $(p=0.045$ ) increasing of task performances execution during AA On condition.

\section{Behavioural Performance Assessment}

The $t$-test results showed that performances of operators during the AA On condition were significantly $(p=0.045)$ higher (WMRT index lower) than performances in the AA Off condition (Figure 4B). Of course, higher reaction times reflect lower performances, and vice versa.

\section{Neurophysiological Workload Analysis}

The $t$-tests showed a significant increasing $(p=0.03)$ of the $\mathrm{W}_{E E G}$ indexes distribution between the Easy and the Hard periods only for the $A A O f f$ condition. On the contrary no significant differences $(p=0.65)$ have been highlighted between the $\mathrm{W}_{E E G}$ indexes related to the Easy and the Hard slots during the $A A$ $O n$ condition (Figure 5A). In addition, a significant increment $(p=0.04)$ of the $\mathrm{W}_{E E G}$ indexes distributions related to the Hard slot of the AA Off condition with respect to the Hard slot of the $A A$ On condition has been reported. In this regard, Figure 5B shows the shape of the $\mathrm{W}_{E E G}$ distributions related to the Hard slot, for both the two conditions (AA On/Off). Instead, no significant trends $(p=0.95)$ have been highlighted between the Easy slots of the two AA conditions. In conclusion, Figure 5C shows the time course of the $\mathrm{W}_{E E G}$ index related to the Easy and Hard slots, in both the two conditions ( $A A$ On/Off) together with the AA activation segments (Trigger) for a representative subject. The figure suggests that when the $\mathrm{AA}$ is activated, the $\mathrm{W}_{E E G}$ index related to the $A A$ On condition decreases accordingly.

In conclusion, the $t$-test results showed that the number of AA activations triggered by the $\mathrm{W}_{E E G}$ index ( $A A O n$ condition) was significantly lower $(p=0.04)$ during the Easy period with respect to the Hard one. In other words, the classifier triggered more often the ATM interface when the operator's workload was classified as HIGH (i.e., during the Hard period).

\section{DISCUSSION}

In the present study, we proposed a $\mathrm{pBCI}$ system able to evaluate and classify online the operators' mental workload by using the EEG activity $\left(\mathrm{W}_{E E G}\right)$. Depending on the classification result (LOW or HIGH), the system was able to trigger online the operator's interface, changing its behavior by activating or deactivating Adaptive Automation (AA) solutions. The system has been integrated in an already existing ATM experimental platform, developed at ENAC. Twelve ATCO students have been asked to test the system by managing high realistic ATM scenarios under different difficulty levels. In particular, we expected that the proposed system was able: (i) to trigger in the right way the ATM interface (i.e., the number of times in which the system activates the AA should be higher during the Hard scenario period in respect to the Easy one in order to prevent overload situation during the former and underload during the latter), and (ii) to induce a decreasing of the mental workload perceived by the operators when the adaptive solutions were activated and consequently an increasing in task performances execution.

Regarding the first point, results confirmed that the number of AA activations were significantly $(\mathrm{p}=0.04)$ higher during the Hard scenario period with respect to the easy one. The behavior of activating AA solutions only when the workload of the operator becomes high is an important issue. In fact, if the AA solutions were improperly or even always activated, the workload of the user could decrease too much, and, in general, induce performance reduction and decreasing safety. In this regard, results showed no differences in terms of mental workload between the Easy scenarios related to the AA On and AA Off conditions.

For the second point, results revealed a significant decreasing of the mental workload of the operator when the proposed $\mathrm{pBCI}$ system activated the AA solutions (AA On condition) with respect to the condition without AA solutions ( $A A$ Off). Furthermore, in the $A A$ On condition, the EEG-based mental workload index $\left(\mathrm{W}_{E E G}\right)$ related to the Hard task was not significantly higher than the Easy related workload condition. On the contrary, when the AA solutions were not activated (AA Off condition), the Hard related mental workload was significantly 


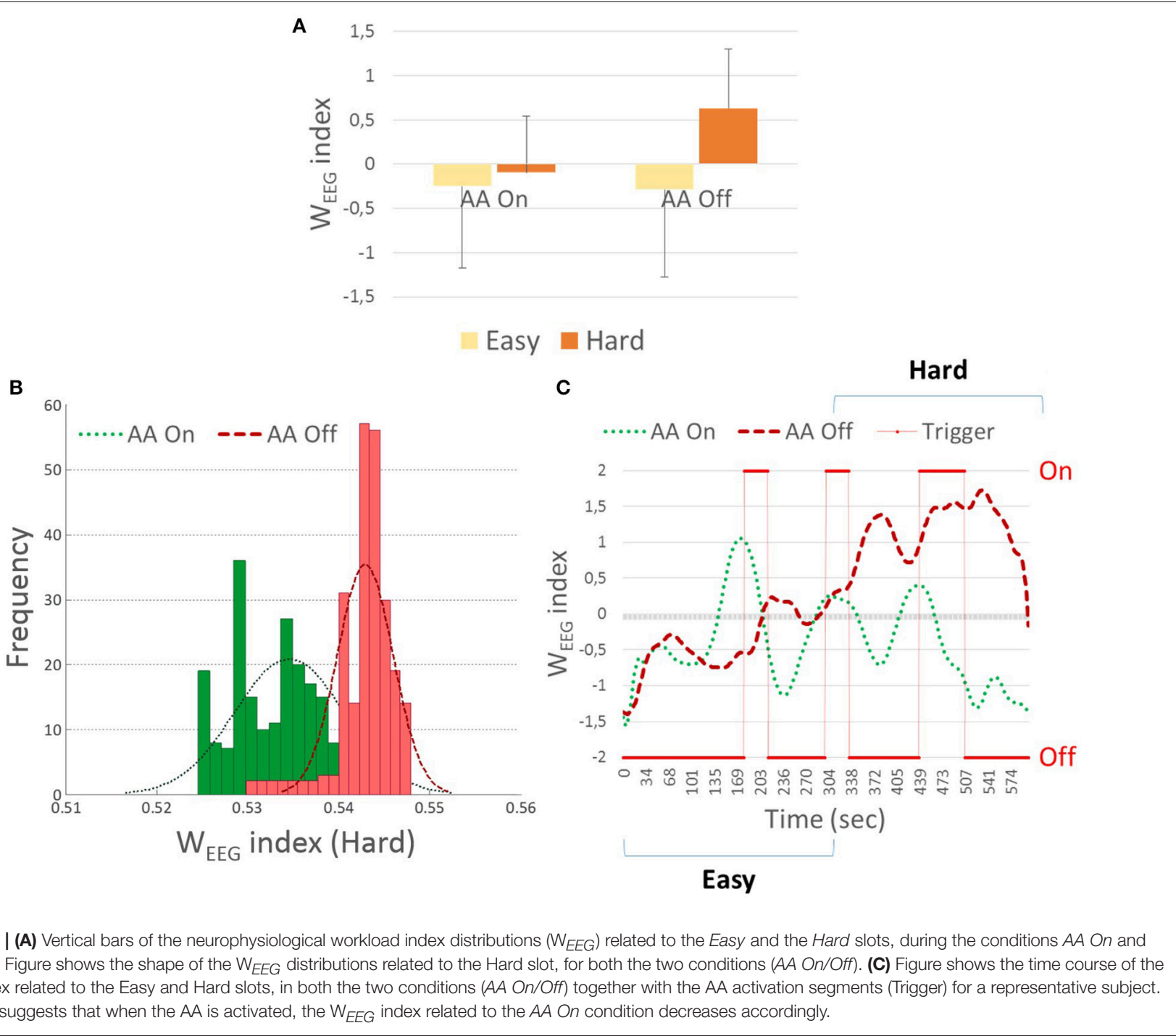

higher than the Easy one. This behavior was confirmed also by the subjective measures, in particular, the NASA-TLX questionnaire revealed an increasing of the perceived mental workload when the AA solutions were not activated $(A A O f f)$ with respect to the scenarios in which the $\mathrm{pBCI}$ could activate the AA solutions $(A A O n)$. Finally, behavioral performance analysis also revealed that AA On condition induced a significant increment in task performance with respect to the AA Off condition.

The results achieved in this study, performed in a highrealistic setting, confirmed the findings of other similar works. For example, Freeman et al. (1999) have proposed an application consisting in the switching between automatic and manual mode of the tracking task of the Multiple-Attribute Task Battery (MATB, Comstock, 1994) by adopting EEG indexes based on the Theta and Alpha band power spectra over the parietal cortex. A similar study was also proposed by Prinzel et al. (2000). Another application was performed by Berka et al. (2005) by using a military simulation environment triggered by an EEG-based workload index. All the studies have highlighted a significant decrement in the mental workload experienced by the users by activating the automation solutions. Anyhow, it has to be stressed that such studies have been performed in laboratory settings, where the environment and the difficulty levels used in the proposed tasks were very under control.

On the contrary, in the present study, we confirmed the same findings, but in realistic settings where difficulty levels were not constant but changed over time reflecting real traffic scenarios, and the operators could communicate and move as they normally do during real work shifts. In conclusion, despite the possible presence of artifacts coupled to the EEG signal, and the realistic shape of the traffic samples, the significance of the results is remarkable.

The only study performed in a more realistic environment was proposed by Abbass et al. (2014), in which four ATC experts had to manage a simulator for $50 \mathrm{~min}$, while both EEG and traffic indicators were used in a rule-based system, which decided 
if there was the need to activate adaptation (AA) or not. In particular, the EEG index was based on the Theta to Beta ratio over the whole brain scalp. The results indicated that the 4 subjects perceived an overall increasing in their performance (assessed by questionnaire) when the AA was enabled, but this result was not statistically significant. In addition, no significant differences have been highlighted between complexity indexes (estimated from task parameters) when the AA was enabled or disabled.

\section{CONCLUSION}

The aim of the study was to investigate the possibility of using information coming from the operators' brain activity (i.e., mental workload) to realize a p-BCI system able to trigger specific Adaptive Automation solutions in Air Traffic Management contexts. The results demonstrated that the proposed pBCI system was able to (i) differentiate workload levels related to different difficulty tasks (i.e., Easy and Hard), (ii) trigger the AA solutions mostly when the workload of the operator was high, so preventing overload and underload situations. Also, it has been demonstrated that the whole AA system was able to (iii) induce a significant reduction of the workload level experienced by the operator during the execution of the ATM task and iv) a significant increasing of the task performance execution.

Thanks to the promising results, further experiments will be performed to investigate the possibility to develop AA solutions triggered by using more than two states (i.e., workload HIGH and LOW), in order to have a more specific Dynamic Function Allocation. Ideally, it should be possible to modulate all the 10

\section{REFERENCES}

Abbass, H. A., Tang, J., Amin, R., Ellejmi, M., and Kirby, S. (2014). Augmented cognition using Real-time EEG-based adaptive strategies for air traffic control. Proc. Hum. Factors Ergon. Soc. Annu. Meet. 58, 230-234. doi: 10.1177/154193 1214581048

Aloise, F., Aricò, P., Schettini, F., Riccio, A., Salinari, S., Mattia, D., et al. (2012). A covert attention P300-based brain-computer interface: geospell. Ergonomics 55, 538-551. doi: 10.1080/00140139.2012.661084

Aloise, F., Schettini, F., Aricò, P., Bianchi, L., Riccio, A., Mecella, M., et al. (2010). "Advanced brain computer interface for communication and control," in Proceedings of the International Conference on Advanced Visual Interfaces, AVI '10. (New York, NY: ACM), 399-400.

Aricò, P., Aloise, F., Schettini, F., Riccio, A., Salinari, S., Babiloni, F., et al. (2011). Geospell: an alternative p300-based speller interface towards no eye gaze require. Int. J. Bioelectromagn. 13, 152-153.

Aricò, P., Borghini, G., Di Flumeri, G., and Babiloni, F. (2015a). Metodo Per Stimare Uno Stato Mentale, in Particolare Un Carico Di Lavoro, e Relativo Apparato (A Method for the Estimation of Mental State, in Particular of the Mental Workload and its Device). P1108IT00.

Aricò, P., Borghini, G., Di Flumeri, G., Colosimo, A., Graziani, I., Imbert, J. P., et al. (2015b). Reliability over time of EEG-based mental workload evaluation during Air Traffic Management (ATM) tasks. Conf Proc IEEE Eng Med Biol Soc. 2015, 7242-7245. doi: 10.1109/EMBC.2015.7320063

Aricò, P., Borghini, G., Di Flumeri, G., Colosimo, A., Pozzi, S., and Babiloni, F. (2016). A passive Brain-Computer Interface (p-BCI) application for the mental workload assessment on professional Air Traffic Controllers (ATCOs) during realistic ATC tasks. Prog. Brain Res. 228, 295-328. doi: 10.1016/bs.pbr.2016.04.021 levels of LOA (Sheridan and Verplank, 1978) depending on the actual mental workload of the user.

\section{AUTHOR CONTRIBUTIONS}

PA wrote the paper and developed part of the online system to measure the mental workload of the user. In addition he took part to the definition of the experimental protocol. GB and GD made offline data analysis and related statistics. In addition they contributed to the writing process and to the protocol definition and data acquisition. AC contributed to the revision of the manuscript. SB, AG, and SP defined the human factor concepts at the basis of the adaptive automation. In addition they contributed to the definition of the experimental protocol. JI, GG, and RB developed the adaptive solutions used in the experiments and implemented the connection between the ATM interface and the EEG-based workload index. FB supervised and contributed to the definition of the methodologies used in the actual work and revised the entire manuscript.

\section{ACKNOWLEDGMENTS}

This work is co-financed by the European Commission by Horizon2020 projects Sesar-01-2015 Automation in ATM, "Stress," GA n. 699381 and Sesar-06-2015 High Performing Airport Operations, "MOTO," GA n. 699379. The grant provided by the Italian Minister of University and Education under the PRIN 2012, GA n. 2012WAANZJ scheme to FB. is also gratefully acknowledged.

Aricò, P., Borghini, G., Graziani, I., Bianchini, F., Cincotti, F., and Babiloni, F. (2013). A brain computer interface system for the online evaluation of ATCs' workload. Ital. J. Aerosp. Med.

Aricò, P., Borghini, G., Graziani, I., Imbert, J. P., Granger, G., Benhacene, R., et al. $(2015 \mathrm{c})$. ATCO: neurophysiological analysis of the training and of the workload. Ital. J. Aerosp. Med. 12, 18-34.

Aricò, P., Borghini, G., Graziani, I., Taya, F., Sun, Y., Bezerianos, A., et al. (2014). "Towards a multimodal bioelectrical framework for the online mental workload evaluation," in Presented at the 36th Annual International Conference of the IEEE Engineering in Medicine and Biology Society, (Chicago, IL).

Babiloni, F., Carducci, F., Cincotti, F., Del Gratta, C., Roberti, G. M., Romani, G. L., et al. (2000). Integration of high resolution EEG and functional magnetic resonance in the study of human movement-related potentials. Methods Inf. Med. 39, 179-182.

Bamber, D. (1975). The area above the ordinal dominance graph and the area below the receiver operating characteristic graph. J. Math. Psychol. 12, 387-415. doi: 10.1016/0022-2496(75)90001-2

Berka, C., Levendowski, D. J., Ramsey, C. K., Davis, G., Lumicao, M. N., Stanney, K., et al. (2005). "Evaluation of an EEG workload model in an Aegis simulation environment," in Presented at the Defense and Security, International Society for Optics and Photonics (Orlando, FL), 90-99.

Blankertz, B., Tangermann, M., Vidaurre, C., Fazli, S., Sannelli, C., Haufe, S., et al. (2010). The berlin brain-computer interface: non-medical uses of BCI technology. Front. Neurosci. 4:198. doi: 10.3389/fnins.2010.00198

Borghini, G., Arico, P., Astolfi, L., Toppi, J., Cincotti, F., Mattia, D., et al. (2013). Frontal EEG theta changes assess the training improvements of novices in flight simulation tasks. Conf. Proc. IEEE Eng. Med. Biol. Soc. 2013, 6619-6622. doi: 10.1109/EMBC.2013.6611073 
Borghini, G., Aricò, P., Di Flumeri, G., Salinari, S., Colosimo, A., Bonelli, S., et al. (2015a). Avionic technology testing by using a cognitive neurometric index: a study with professional helicopter pilots. Conf. Proc. IEEE Eng. Med. Biol. Soc. 2015, 6182-6185. doi: 10.1109/embc.2015.7319804

Borghini, G., Aricò, P., Ferri, F., Graziani, I., Pozzi, S., Napoletano, L., et al. (2014). A neurophysiological training evaluation metric for air traffic management. Conf. Proc. IEEE Eng. Med. Biol. Soc. 2014, 3005-3008. doi: 10.1109/EMBC.2014.6944255

Borghini, G., Aricò, P., Graziani, I., Salinari, S., Sun, Y., Taya, F., et al. (2015b). Quantitative assessment of the training improvement in a motor-cognitive task by using EEG, ECG and EOG signals. Brain Topogr. 29, 149-161. doi: 10.1007/s10548-015-0425-7

Borghini, G., Astolfi, L., Vecchiato, G., Mattia, D., and Babiloni, F. (2012). Measuring neurophysiological signals in aircraft pilots and car drivers for the assessment of mental workload, fatigue and drowsiness. Neurosci. Biobehav. Rev. 44, 58-75. doi: 10.1016/j.neubiorev.2012.10.003

Brookings, J. B., Wilson, G. F., and Swain, C. R. (1996). Psychophysiological responses to changes in workload during simulated air traffic control. Biol. Psychol. 42, 361-377.

Byrne, E. A., and Parasuraman, R. (1996). Psychophysiology and adaptive automation. Biol. Psychol. 42, 249-268. doi: 10.1016/0301-0511(95)05161-9

Calabrese, E. J. (2008). Neuroscience and hormesis: overview and general findings. Crit. Rev. Toxicol. 38, 249-252. doi: 10.1080/10408440801981957

Comstock, J. R. (1994). MATB - Multi-Attribute Task Battery for Human Operator Workload and Strategic Behavior Research. Washington, DC: National Aeronautics and Space Administration.

Craven, P. L., Belov, N., Tremoulet, P., Thomas, M., Berka, C., Levendowski, D., et al. (2006). "Cognitive workload gauge develpment: comparison of Real-time classification methods," in Past, Present and Future, Foundations of Augmented Cognition (Arlington, TX: Strategic Analysis, Inc.), 66-74.

Delorme, A., and Makeig, S. (2004). EEGLAB: an open source toolbox for analysis of single-trial EEG dynamics including independent component analysis. J. Neurosci. Methods 134, 9-21. doi: 10.1016/j.jneumeth.2003.10.009

Di Flumeri, G., Aricò, P., Borghini, G., Colosimo, A., and Babiloni, F. (in press). A new regression-based method for the eye blinks artifacts correction in the EEG signal, without using any EOG channel. Conf. Proc. IEEE Eng. Med. Biol. Soc.

Di Flumeri, G., Borghini, G., Aricò, P., Colosimo, A., Pozzi, S., Bonelli, S., et al. (2015). "On the use of cognitive neurometric indexes in aeronautic and air traffic management environments," in Symbiotic Interaction, eds B, Blankertz, G. Jacucci, L. Gamberini, A. Spagnolli, and J. Freeman (Cham: Springer International Publishing), 45-56.

Dorneich, M. C., Ververs, P. M., Mathan, S., and Whitlow, S. D. (2005). "A joint human-automation cognitive system to support rapid decision-making in hostile environments," in 2005 IEEE International Conference on Systems, Man and Cybernetics. Presented at the 2005 IEEE International Conference on Systems, Man and Cybernetics, Vol. 3 (Piscataway, NJ: IEEE), 2390-2395.

Draper, N. R. (1998). Applied regression analysis. Commun. Stat. Theory Methods 27, 2581-2623. doi: 10.1080/03610929808832244

Fawcett, T. (2006). An Introduction to ROC Analysis. Pattern Recogn. Lett. 27, 861-874. doi: 10.1016/j.patrec.2005.10.010

Freeman, F. G., Mikulka, P. J., Prinzel, L. J., and Scerbo, M. W. (1999). Evaluation of an adaptive automation system using three EEG indices with a visual tracking task. Biol. Psychol. 50, 61-76. doi: 10.1016/S0301-0511(99)00002-2

Gevins, A., Smith, M. E., Leong, H., McEvoy, L., Whitfield, S., Du, R., et al. (1998). Monitoring working memory load during computer-based tasks with EEG pattern recognition methods. Hum. Factors J. Hum. Factors Ergon. Soc. 40, 79-91. doi: 10.1518/001872098779480578

Gratton, G., Coles, M. G., and Donchin, E. (1983). A new method for off-line removal of ocular artifact. Electroencephalogr. Clin. Neurophysiol. 55, 468-484. doi: 10.1016/0013-4694(83)90135-9

Hancock, P. A., Jagacinski, R. J., Parasuraman, R., Wickens, C. D., Wilson, G. F., and Kaber, D. B. (2013). Human-Automation interaction research past, present, and future. Ergon. Des. Q. Hum. Factors Appl. 21, 9-14. doi: $10.1177 / 1064804613477099$

Hancock, P. A., and Warm, J. S. (1989). A dynamic model of stress and sustained attention. Hum. Factors J. Hum. Factors Ergon. Soc. 31, 519-537.

Hart, S. G., and Staveland, L. E. (1988). "Development of NASA-TLX (Task Load Index): results of empirical and theoretical research," in Human Mental
Workload, eds P. A. Hancock and N. Meshkati (Amsterdam: North-Holland Press).

Hilburn, B., Jorna, P. G., Byrne, E. A., and Parasuraman, R. (1997). "The effect of adaptive air traffic control (ATC) decision aiding on con- troller mental workload," in Human-Automation Interaction: Research and Practice, eds M. Mouloua and J. Koonce (Mahwah, NJ: Erlbaum), 84-91.

Jurcak, V., Tsuzuki, D., and Dan, I. (2007). 10/20, 10/10, and 10/5 systems revisited: their validity as relative head-surface-based positioning systems. Neuroimage 34, 1600-1611. doi: 10.1016/j.neuroimage.2006.09.024

Klimesch, W. (1999). EEG alpha and theta oscillations reflect cognitive and memory performance: a review and analysis. Brain Res. Brain Res. Rev. 29, 169-195. doi: 10.1016/S0165-0173(98)00056-3

Kohlmorgen, J., Dornhege, G., Braun, M. L., Blankertz, B., Müller, K.-R., Curio, G., et al. (2007). "Improving human performance in a real operating environment through real-time mental workload detection," in Toward Brain-Computer Interfacing, eds G. Dornhege, J. del R. Millán, T. Hinterberger, D. McFarland, and K.-R. Müller (Cambridge, MA: MIT Press), 409-422.

McLachlan, G., Do, K.-A., and Ambroise, C. (2005). Analyzing Microarray Gene Expression Data. Hoboken, NJ: John Wiley \& Sons.

Mühl, C., Jeunet, C., and Lotte, F. (2014). EEG-based workload estimation across affective contexts. Front. Neurosci. 8:114. doi: 10.3389/fnins.2014.00114

O'Donnell, R. D., and Eggemeier, F. T. (1986). "Workload assessment methodology," in Handbook of Perception and Human Performance, Volume 2. Cognitive Processes and Performance, eds K. R. Boff, L. Kaufman, and J. P. Thomas (New York, NY: John Wiley and Sons, Inc.), 42-1-42-49.

Parasuraman, R., Bahri, T., Deaton, J. E., Morrison, J. G., and Barnes, M. (1992). Theory and Design of Adaptive Automation in Aviation Systems. Warminster, PA: Naval Air Warfare Center; Aircraft Division.

Parasuraman, R., Molloy, R., and Singh, I. (1993). Performance consequences of automation-induced "complacency". Int. J. Aviat. Psychol. 3, 1-23.

Parsons, H. M. (1985). Automation and the Individual: comprehensive and comparative views. Hum. Factors J. Hum. Factors Ergon. Soc. 27, 99-111.

Pope, A. T., Bogart, E. H., and Bartolome, D. S. (1995). Biocybernetic system evaluates indices of operator engagement in automated task. Biol. Psychol. 40, 187-195. doi: 10.1016/0301-0511(95)05116-3

Prinzel, L. J., Freeman, F. G., Scerbo, M. W., Mikulka, P. J., and Pope, A. T. (2000). A closed-loop system for examining psychophysiological measures for adaptive task allocation. Int. J. Aviat. Psychol. 10, 393-410. doi: 10.1207/S15327108IJAP1004_6

Ramnani, N., and Owen, A. M. (2004). Anterior prefrontal cortex: insights into function from anatomy and neuroimaging. Nat. Rev. Neurosci. 5, 184-194. doi: $10.1038 / \mathrm{nrn} 1343$

Reason, J. (2000). Human error. West. J. Med. 172, 393-396. doi: 10.1136/ewjm.172.6.393

Riccio, A., Holz, E. M., Aricò, P., Leotta, F., Aloise, F., Desideri, L., et al. (2015). Hybrid P300-based brain-computer interface to improve usability for people with severe motor disability: electromyographic signals for error correction during a spelling task. Arch. Phys. Med. Rehabil. 96, S54-S61. doi: 10.1016/j.apmr.2014.05.029

Rouse, W. B. (1976). "Adaptive allocation of decision making responsibility between supervisor and computer," in Monitoring Behavior and Supervisory Control, NATO Conference Series, eds T. B. Sheridan, T.B and G. Johannsen (New York, NY: Plenum). 295-306.

Rouse, W. B. (1988). Adaptive aiding for human/computer control. Hum. Factors J. Hum. Factors Ergon. Soc. 30, 431-443.

Scerbo, M. W. (1996). "Theoretical perspectives on adaptive automation," in Automation and Human Performance: Theory and Applications, Human Factors in Transportation, eds R. Parasuraman, M. Mouloua (Hillsdale, NJ, England: Lawrence Erlbaum Associates, Inc), 37-63.

Scerbo, M. W., Freeman, F. G., and Mikulka, P. J. (2003). A brain-based system for adaptive automation. Theor. Issues Ergon. Sci. 4, 200-219. doi: $10.1080 / 1463922021000020891$

Scerbo, M. W., Freeman, F. G., Mikulka, P. J., Parasuraman, R., Di Nocero, F., and Prinzel, L. J. III. (2001). The Efficacy of Psychophysiological Measures for Implementing Adaptive Technology (NASA/TP-2001-211018). Washington, DC: National Aeronautics and Space Administration.

Schettini, F., Riccio, A., Simione, L., Liberati, G., Caruso, M., Frasca, V., et al. (2015). Assistive device with conventional, alternative, and brain-computer 
interface inputs to enhance interaction with the environment for people with amyotrophic lateral sclerosis: a feasibility and usability study. Arch. Phys. Med. Rehabil. 96, S46-S53. doi: 10.1016/j.apmr.2014.05.027

Schmorrow, D., Stanney, K., Wilson, G., and Young, P. (2006). "Augmented cognition in human-system interaction," in Handbook of Human Factors and Ergonomics, 3rd Edn., ed G. Salvendy (Hoboken, NJ: Wiley), 1364-1384.

Sheridan, T. B. (1992). Telerobotics, Automation, and Human Supervisory Control. Cambridge: MIT Press.

Sheridan, T. B., and Verplank, W. L. (1978). Human and Computer Control of Undersea Teleoperators. Technical Report, Cambridge, MA.

Shou, G., Ding, L., and Dasari, D. (2012). Probing neural activations from continuous EEG in a real-world task: time-frequency independent component analysis. J. Neurosci. Methods 209, 22-34. doi: 10.1016/j.jneumeth.2012.05.022

Toppi, J., Borghini, G., Petti, M., He, E. J., Giusti, V. D., He, B., et al. (2016). Investigating cooperative behavior in ecological settings: an EEG hyperscanning study. PLOS ONE 11:e0154236. doi: 10.1371/journal.pone. 0154236

Vapnik, V. N. (2000). The Nature of Statistical Learning Theory. New York, NY, Springer.

von Luxburg, U., and Schílkopf, B. (2011). "Statistical learning theory: models, concepts, and results," in Handbook for the History of Logic, Vol. 10: Inductive Logic, eds S. H. D. Gabbay and J. Woods (Elsevier).

Wickens, C. D. (1992). Engineering Psychology and Human Performance, 2nd Edn., New York, NY, HarperCollins Publishers.

Wolpaw, J., and Wolpaw, E. W. (2012). Brain-Computer Interfaces: Principles and Practice. Oxford: Oxford University Press.

Wood, J. N., and Grafman, J. (2003). Human prefrontal cortex: processing and representational perspectives. Nat. Rev. Neurosci. 4, 139-147. doi: $10.1038 / \mathrm{nrn} 1033$
Yerkes, R. M., and Dodson, J. D. (1908). The relation of strength of stimulus to rapidity of habit-formation. J. Comp. Neurol. Psychol. 18, 459-482. doi: 10.1002/cne.920180503

Zander, T. O., and Jatzev, S. (2012). Context-aware brain-computer interfaces: exploring the information space of user, technical system and environment. J. Neural Eng. 9, 16003. doi: 10.1088/1741-2560/9/1/016003

Zander, T. O., Kothe, C., Welke, S., and Roetting, M. (2009). "Utilizing Secondary Input from Passive Brain-Computer Interfaces for Enhancing Human-Machine Interaction," in Foundations of Augmented Cognition. Neuroergonomics and Operational Neuroscience. eds D. D. Schmorrow, I. V. Estabrooke, and M. Grootjen (Berlin, Heidelberg: Springer Berlin Heidelberg), 759-771.

Zhang, J. H., Chung, T. D., and Oldenburg, K. (1999). A simple statistical parameter for use in evaluation and validation of high throughput screening assays. J. Biomol. Screen. 4, 67-73. doi: 10.1177/108705719900 400206

Conflict of Interest Statement: The authors declare that the research was conducted in the absence of any commercial or financial relationships that could be construed as a potential conflict of interest.

Copyright (๑) 2016 Aricò, Borghini, Di Flumeri, Colosimo, Bonelli, Golfetti, Pozzi, Imbert, Granger, Benhacene and Babiloni. This is an open-access article distributed under the terms of the Creative Commons Attribution License (CC BY). The use, distribution or reproduction in other forums is permitted, provided the original author(s) or licensor are credited and that the original publication in this journal is cited, in accordance with accepted academic practice. No use, distribution or reproduction is permitted which does not comply with these terms. 